are meant to be used on healthy skin, and this could have been emphasized a little more, particularly with reference to irritancy. On p. 260 the use of "soil or other solid matter" as a test inoculum is not recommended, but in view of her experience of a laboratory contaminant, lower down the page, I feel that soil, shelf dust, hay dust, etc., may be a wise adjunct to the more systematic testing.

The article "Contemporary Trends in Heat Sterilisation" is not so good as the rest of the book. The first eight pages consist of snippets of information that are inadoquately digested, and considerable reliance on a review by Rahn (1945) seems to imply that Rahn was responsible for the work cited. The reference to super-saturated steam (p. 277) is incorrect, so is the reference to metals having a high specific heat (p. 294), and a number of erroneous impressions are also conveyed which may bo the result of poor phrasing, as with the dryness fraction which would not normally have a value greater than one if ( 1 - wetness fraction) is intended.

On p. 278, Fig. 3 gives no units for the abscissa and, if these are $\mathrm{lb} . / \mathrm{in}^{2}$ in excess of atmospheric pressure, the temperatures and pressures are not correlated. Certainly this figure does not appear to refer to dryness fractions as implied in the text.

The occasional inclusion of some elementary practical detail, such as the description of calorimetry (p. 279), is unnecessary and out of keeping with the book as a whole. The main part of the chapter is a detailed description of the design of autoclaves and controls which are now available, and as such scarcely justifies the word 'trends" in the title. Section XXXIII, entitled "Heat Output", rather surprisingly refers to lagging, ventilation and servicing.

The editors must accept some responsibility for not maintaining the standard set by the preceding chapters, and it is to be hoped that in future volumes we shall see more 'appraisals' of the standard set by the authors of the three other sections.

E. SHOTTON

\section{NERVOUS IMPULSE}

\section{The Conduction of the Nervous Impulse}

By Prof. A. L. Hodgkin. (The Sherrington Lectures, VII.) Pp. 108. (Liverpool: The University Press, 1964.) n.p.

HE nervous impulse, how it is generated at a local site and how it is conducted along the nerve fibre, was the subject of the Sherrington Lectures given in 1961 by Prof. A. L. Hodgkin. These lectures, completed with an account of more recent work, have now been published in the form of a book. Hodgkin, working in the Physiology Laboratory at the University of Cambridgo, is Foulerton Research Professor of the Royal Society and received the Nobel Prize in 1963.

Signals conducted along nerve fibres are transmitted as electrical events, as a rule as all-or-nothing signals with a sharp throshold, and an amplitude which is independent of the stimulus. The most essontial structure for the nerve impulse is the cell membrane, which surrounds the axis cylinder of the nerve fibre. This membrane can be seen in the electron microscope, but not in the light microscope, and is about $10^{-6} \mathrm{~cm}$ thick. The membrane has pormeability properties which are very intriguing because it is clear that these permeability properties are the basis for the nerve impulse. The electrolyte in the axis cylinder contains a high concentration of potassium and a low concentration of sodium, while the external solution contains much sodium and little potassium. The resting nerve membrane is only little permeable to ions, but the permeability to potassium is relatively large compared to that of other ions. The movement of potassium through the membrane results, therefore, in a eurrent of such a direction that it tends to charge the membrane towards the electrochemical equilibrium potential for potassium, and, due to this current, a steady potential difference across the membrane is maintained during rest. The axis cylinder is some $60-90 \mathrm{mV}$ negative relative to the external solution. The permeability to other ions, and the consequent ionic currents, of course also affects the membrane potential at rest, but to a smaller extent.

An analysis of the ionic currents associated with step changes of the membrane potential indicates that the membrane permeability depends on membrane potential and time. When the axis cylinder potential is changed in positive direction from its resting value, then the membrane undergoes an initial transient increase in sodium permeability. The sodium permoability increase is followed by an increase in potassium permeability. Both these permeability changes are continuous smooth functions of membrane potential and of tims, but are independent of sodium and potassium concentrations. The result of complicated and tedious experimental and analytical work is a fairly complete formal description of these permeability changes. A number of empirical constants in a number of equations thus describe the specific ionic currents in the nerve membrane as associated with step changes in membrane potential. The same set of constants and basic equations might be expected to apply also for the condition that the membrane undorgoes a more complicated change. Further, the equations might predict the action potential of the nerve. Numerical solutions indicate indeed that the equations very satisfactorily predict many of the known excitability properties of the nerve fibre.

The impulse mechanism in the nerve, and very likely also in musele, is thus explained quantitatively on the basis of these specific permeability changes in the very thin surface membrane. The ionic currents are passive and are consequences of the permeability changes and the electro-chemical driving forces. Immediate energy requirement for impulse generation is thus available in the form of a concentration battery, that is, the unequal distribution of ions on both sides of the membrane.

The specific ionic membrane currents have also been measured with the aid of radioactive isotopes and the findings support the ionic theory for impulse conduction. While these measurements resolve the ionic fluxes in many ways more clearly than the electrical techniques, the isotope measurements lack a high time resolution. Isotope measurements are also directed towards another goal, to investigate the ionic fluxes against the electrochemical gradient. These fluxes requiro energy, and are ultimately necessary in order to maintain the concentra. tion gradients across the membrane and thereby maintain the energy used during the impulse generation. Energyrich phosphate compounds are evidently used by the nerve for the active ionic transports against the electrochemical gradients.

In the preface of The Conduction of the Nervous Impulse, Prof. Hodgkin writes: "... I talked mainly about experiments on which my colleagues and I had been engaged in the immediate past". When reading the book I found this true. The preface does not tell, however, that Hodgkin and his colleagues have radically changed our concepts of how the impulse is generated in the nerve. Neither is it mentioned that a very complete picture of the "Cambridge Nerve" is given.

The book is written in a straightforward style. Rather drastic simplifications of the basic material have been made, but made in a masterly fashion so that the distortions caused are negligible.

I like the book; without doubt it is the best summary of the nervous impulse I have read. I can warmly recommend it to students within the field, to students in neighbouring fields and to all those who want to obtain an understanding of how the nervous impulse is generated and conducted.
B. Frankenhatuser 